\title{
A CLASS OF RISK PROCESSES WITH DELAYED CLAIMS: RUIN PROBABILITY ESTIMATES UNDER HEAVY TAIL CONDITIONS
}

\author{
AYALVADI GANESH, ${ }^{*}$ Microsoft Research \\ GIOVANNI LUCA TORRISI, ${ }^{* *}$ Istituto per le Applicazioni del Calcolo, Roma
}

\begin{abstract}
We consider a class of risk processes with delayed claims, and we provide ruin probability estimates under heavy tail conditions on the claim size distribution.

Keywords: Extreme value theory; Poisson process; regular variation; renewal process; ruin probability; shot noise process; subexponential distribution

2000 Mathematics Subject Classification: Primary 91B30; 60G55
\end{abstract}

\section{Introduction}

In this paper we are concerned with stochastic processes with drifts of the form

$$
S(t)=\sum_{n \geq 1} F\left(t-T_{n}\right) Z_{n} \mathbf{1}_{(0, t]}\left(T_{n}\right)-c t,
$$

where $\left\{T_{n}\right\}_{n \geq 1}, T_{0}=0$, are the points (epochs) of a renewal process whose interarrival times, $U_{n}=T_{n}-T_{n-1}$, have finite mean, $\left\{Z_{n}\right\}_{n \geq 1}$ is a sequence of independent and identically distributed positive random variables independent of $\left\{T_{n}\right\}_{n \geq 1}, c>0$ is a positive constant, and $F(\cdot)$ is a distribution function (DF) such that $F(t)=0$ for $t<0$.

An important example of the stochastic model considered above arises in insurance risk theory; see Klüppelberg and Mikosch (1995a), (1995b), Mikosch and Nagaev (1998), Brémaud (2000), and Klüppelberg et al. (2003), where Poisson shot noise processes were considered. In Brémaud (2000), large deviations theory was used to give Cramér-Lundberg-type estimates of the infinite-horizon ruin probability. Other models of delayed claims (not based on shot noise processes) were considered by Waters and Papatriandafylou (1985) and, more recently, by Yuen et al. (2005), where a martingale approach was used to estimate the infinite-horizon ruin probability under light tail conditions.

The interpretation of the process $\{S(t)\}_{t \geq 0}$ in the insurance context is as follows. Suppose that claims $\left\{Z_{n}\right\}_{n \geq 1}$ occur according to a renewal process $\left\{T_{n}\right\}_{n \geq 1}$, and that the insurance company honors the claim $Z_{n}$, which occurs at time $T_{n}$, at the rate $f\left(\cdot-T_{n}\right) Z_{n}$, with $f(\cdot)$ being a probability density on $(0, \infty)$. The total claim paid in the time interval $(0, t]$ is then

$$
\sum_{n \geq 1} F\left(t-T_{n}\right) Z_{n} \mathbf{1}_{(0, t]}\left(T_{n}\right),
$$

Received 22 November 2005; revision received 17 August 2006.

* Postal address: Microsoft Research, 7 J J Thomson Avenue, Cambridge CB3 0FB, UK.

Email address: ajg@microsoft.com

** Postal address: Istituto per le Applicazioni del Calcolo 'M. Picone' (IAC-CNR), Viale del Policlinico 137, 00161

Roma, Italia. Email address: torrisi@iac.rm.cnr.it 
where $F(\cdot)$ is the DF with density $f(\cdot)$. If the insurance company receives premium income at constant rate $c>0$, then $S(t)$ as defined above is the excess of claims over premiums. If we assume that the insurance company has an initial capital $u>0$, then we say that ruin occurs at the first time, $t$, that $S(t) \geq u$, if there is such a time. This leads us to define the infinite-horizon ruin probability

$$
\psi(u)=\mathrm{P}\left(\sup _{t \geq 0} S(t) \geq u\right)
$$

and the finite-horizon ruin probability

$$
\psi(u, T)=\mathrm{P}\left(\sup _{t \in[0, T]} S(t) \geq u\right)
$$

where $T>0$ is a positive constant.

In this paper, under heavy tail assumptions on the distribution of $Z_{1}$, we give asymptotic estimates for $\psi(u)$ and $\psi(u, e(u) T)$ as $u \rightarrow \infty$, where $e(u)=\mathrm{E}\left[Z_{1}-u \mid Z_{1}>u\right]$ is the mean excess function of $Z_{1}$. Our results exploit the heavy tail intuition which predicts exceedances of level $u>0$ to occur as the consequence of one big jump. In particular, we show that the classical ruin probability estimate (see Teugels and Veraverbeke (1973), Veraverbeke (1977), and Embrechts and Veraverbeke (1982)) holds unchanged for $\psi(u)$. Likewise, assuming Poisson claim arrivals, we show that some well-known estimates of the finite-horizon ruin probability of the Cramér-Lundberg model (see Asmussen and Klüppelberg (1996)) hold also for $\psi(u, e(u) T)$. This is an insensitivity property of the model considered, in that the asymptotic behavior of $\psi(u)$ and $\psi(u, e(u) T)$ depends only on the distribution of $Z_{1}$, not on the shape or nature of the shot. It is an analogue of an insensitivity property in the light tail case with Poisson claim arrivals (see Brémaud (2000) for the infinite-horizon case and Macci et al. (2005) for the finite-horizon case) where the large deviation rate functions of $\psi(u)$ and $\psi(u, u T)$ do not depend on the shape of the shot. We also give a short proof of the insensitivity property in the light tail case, for completeness.

Our technique is based on a recent work, Albrecher and Asmussen (2006), in which risk processes with shot noise Cox claim arrivals were considered. A closely related work is Asmussen et al. (1999), where the heavy-tailed behavior of the infinite-horizon ruin probability of risk processes with ergodic or regenerative input was studied.

In the literature on shot noise models, one usually deals with shot shapes of the form $h(t, z)$ (in place of the multiplicative form $F(t) z)$, where $h(\cdot, z)$ is a nondecreasing function for each $z$. The extension of the results of this paper to this more general situation is an open problem.

The paper is structured as follows. We recall some preliminaries and introduce some notation in Section 2. Our results on ruin probabilities with heavy-tailed claim sizes are given in Section 3. Finally, we provide ruin probability estimates for light-tailed claim sizes in Appendix A; while these estimates coincide with those of Brémaud (2000), their derivation is simpler.

\section{Preliminaries}

Recall that a DF $G(\cdot)$ is said to be subexponential if its support is $(0, \infty)$ and $\bar{G}^{* 2} \sim 2 \bar{G}$ (see, for instance, Rolski et al. (1999)). Here $\bar{G}=1-G$ denotes the tail of $G, \bar{G}^{* 2}$ (.) denotes the two-fold convolution of $\bar{G}(\cdot)$, and we write $g_{1} \sim g_{2}$ if the functions $g_{1}(\cdot)$ and $g_{2}(\cdot)$ are such that $\lim _{x \rightarrow \infty} g_{1}(x) / g_{2}(x)=1$; we write $g_{1}(x)=o\left(g_{2}(x)\right)$ if $\lim _{x \rightarrow \infty} g_{1}(x) / g_{2}(x)=0$. We say that a positive function $g(\cdot)$ on $(0, \infty)$ is regularly varying at infinity of index $\alpha \in \mathbb{R}$, 
written $g \in \mathcal{R}(\alpha)$, if $g(x) \sim x^{\alpha} L(x)$ as $x \rightarrow \infty$. Here $L(\cdot)$ is a slowly varying function, that is, $\lim _{x \rightarrow \infty} L(t x) / L(x)=1$ for each $t>0$.

The family of subexponential DFs will be denoted by $\&$. It can be further classified using extreme value theory. Goldie and Resnick (1988) showed that if $G \in \&$ satisfies some smoothness conditions, then $G$ belongs to the maximum domain of attraction of either the Fréchet distribution $\Phi_{\alpha}(x)=\mathrm{e}^{-x^{-\alpha}}$ or the Gumbel distribution $\Lambda(x)=\mathrm{e}^{-\mathrm{e}^{-x}}$. Moreover, in the former case, it has a regularly varying tail of index $-\alpha$.

Throughout this paper we denote by $B(\cdot)$ the DF of $Z_{1}$ and by $B_{0}(\cdot)$ its integrated tail DF:

$$
\overline{B_{0}}(u)=\frac{1}{\mu} \int_{u}^{\infty} \bar{B}(x) \mathrm{d} x, \quad u>0, \quad \text { where } \mu=\mathrm{E}\left[Z_{1}\right] .
$$

We assume that $B_{0} \in \&$ and that either $\bar{B} \in \mathcal{R}(-\alpha-1)$ or $B$ belongs to the maximum domain of attraction of the Gumbel distribution, written $B \in \operatorname{MDA}(\Lambda)$. We also assume the classical net profit condition,

$$
\rho=\mu / c v<1, \quad \text { where } v=\mathrm{E}\left[U_{1}\right]
$$

to hold. This condition says that the mean rate, $c$, at which premium income is earned exceeds the mean rate, $\mu / \nu$, at which claims need to be paid out. If this condition does not hold, then ruin is certain.

\section{Ruin probabilities}

In this section we derive asymptotic estimates for $\psi(u)$ and $\psi(u, e(u) T)$ as $u \rightarrow \infty$, under heavy tail conditions on $Z_{1}$. To this end, we compare the process $\{S(t)\}_{t \geq 0}$ defined in (1.1) with the process $\{C(t)\}_{t \geq 0}$ given by

$$
C(t)=\sum_{n \geq 1} Z_{n} \mathbf{1}_{(0, t]}\left(T_{n}\right)-c t .
$$

Clearly, the following domination holds:

$$
S(t) \leq C(t) \quad \text { almost surely, for all } t \geq 0 .
$$

We shall use this to obtain upper bounds on the ruin probabilities in both the infinite- and finitehorizon settings. Lower bounds will be obtained by comparing $S(\cdot)$ with a different classical risk process.

\subsection{The infinite-horizon case}

Let $\Psi(\cdot)$ denote the infinite-horizon ruin probability for the risk process $\{C(t)\}_{t \geq 0}$. By (3.2), $\psi(u) \leq \Psi(u)$ for all $u>0$. Therefore, by the classical ruin estimate (see Teugels and Veraverbeke (1973), Veraverbeke (1977), and Embrechts and Veraverbeke (1982); see also Theorem 6.5.11 of Rolski et al. (1999)), for an arbitrary $B_{0} \in \&$ we have

$$
\limsup _{u \rightarrow \infty} \frac{\psi(u)}{\overline{B_{0}}(u)} \leq \lim _{u \rightarrow \infty} \frac{\Psi(u)}{\overline{B_{0}}(u)}=\frac{\rho}{1-\rho} .
$$

We shall obtain matching lower bounds, adapting to our context the techniques of Albrecher and Asmussen (2006). We begin by bounding the risk process $S(\cdot)$ from below. For all $t \geq 0$ and $a>0$, define the risk processes

$$
\check{S}_{a}(t)=\sum_{n \geq 1} F(a) Z_{n} \mathbf{1}_{(0, t]}\left(T_{n}\right)-c t
$$


and

$$
\check{C}_{a}(t)=\sum_{n \geq 1} Z_{n} \mathbf{1}_{(0, t]}\left(T_{n}\right)-\frac{c}{F(a)} t .
$$

It is clear from the monotonicity of $F(\cdot)$ that

$$
S(t) \geq \check{S}_{a}(t-a)-c a \text { almost surely, for all } t>a .
$$

Therefore, letting $\check{\Psi}_{a}(\cdot)$ denote the infinite-horizon ruin probability relative to the risk process $\left\{\check{C}_{a}(t)\right\}_{t \geq 0}$, we obtain

$$
\psi(u) \geq \check{\Psi}_{a}((u+c a) / F(a)) \text { for all } u>0 .
$$

We shall use this to obtain asymptotic lower bounds on the ruin probability matching the upper bound in (3.3) under additional conditions on the claim size distribution.

Our first result states that if the claim sizes have regularly varying tails, then the ruin probability with delayed claims is insensitive to the shape of the shot, in the sense that it is asymptotically equivalent to the ruin probability in the classical model. We state this more precisely as follows.

Proposition 3.1. If $\bar{B} \in \mathcal{R}(-\alpha-1)$ for some $\alpha>0$, then

$$
\lim _{u \rightarrow \infty} \frac{\psi(u)}{\overline{B_{0}}(u)}=\frac{\rho}{1-\rho} .
$$

If the claim size distribution has lighter tails, then we need additional assumptions on the shape of the shot in order to retain asymptotic equivalence with the classical model. The first part of our next result shows that if the shot has compact support, then (3.7) holds so long as the claim size distribution has an integrated tail which is subexponential. The second part shows that if the tail of the shot decays sufficiently rapidly relative to the mean excess function of the claim size distribution, then (3.7) continues to hold.

Proposition 3.2. (a) If $B_{0} \in \delta$ and $F(\cdot)$ has compact support, then (3.7) holds.

(b) Suppose that $B \in \operatorname{MDA}(\Lambda), B_{0} \in \delta$, and that e $(u) \sim g(u)$ as $u \rightarrow \infty$, for some eventually nondecreasing function $g(\cdot)$. Suppose further that there is a $\gamma>0$ such that

$$
u \bar{F}\left(u^{1 / \gamma}\right)=o(e(u))
$$

and

$$
u^{1 / \gamma}=o(e(u))
$$

as $u \rightarrow \infty$. Then (3.7) holds.

Before proving Propositions 3.1 and 3.2, we work out some examples of the conditions imposed on the shot shape, under part (b) of Proposition 3.2, by some heavy-tailed distributions of practical interest. These examples show that the assumptions of part (b) are not too restrictive.

Example 3.1. (Weibull distribution.) Suppose that the claim size distribution has tail $\bar{B}(u)=$ $\mathrm{e}^{-u^{\alpha}}$ for $u \geq 0$, where $\alpha \in(0,1)$ is the shape parameter of the Weibull distribution. It is well known that $B \in \operatorname{MDA}(\Lambda)$ and $B_{0} \in \&$ (see, for instance, Embrechts et al. (1997)). Moreover, by partial integration we obtain

$$
e(u) \sim \frac{u^{1-\alpha}}{\alpha} \quad \text { as } u \rightarrow \infty
$$


therefore, $e(u)$ is asymptotically equivalent to a nondecreasing function. Now, (3.8) can be rewritten as $\bar{F}(u)=o\left(e\left(u^{\gamma}\right) / u^{\gamma}\right)=o\left(u^{-\gamma \alpha}\right)$. Hence, (3.8) and (3.9) hold provided that there is a $\gamma>1 /(1-\alpha)$ such that $\bar{F}(u)=o\left(u^{-\gamma \alpha}\right)$. In particular, this is the case if $F$ is either light tailed or has a regularly varying tail of index $-\beta, \beta>\alpha /(1-\alpha)$.

Example 3.2. (Lognormal distribution.) Denote by $\Phi(\cdot)$ the DF of the standard normal distribution and take $B(u)=\Phi((\ln u-\omega) / \sigma), u>0$, where $\omega \in \mathbb{R}$ and $\sigma>0$ are given constants. It is well known that $B \in \operatorname{MDA}(\Lambda)$ and $B_{0} \in \delta$ (see, for instance, Asmussen and Klüppelberg (1996)). Furthermore, using Mill's ratio and l'Hôpital's rule, we obtain

$$
e(u) \sim \frac{\sigma^{2} u}{\ln u-\omega} \quad \text { as } u \rightarrow \infty .
$$

Hence, $e(u)$ is asymptotically equivalent to an eventually nondecreasing function, and (3.9) is satisfied for any $\gamma>1$. Also, (3.8) is satisfied if $\bar{F}\left(u^{1 / \gamma}\right)=o(1 / \ln u)$, that is, $\bar{F}(u)=o(1 / \ln u)$ irrespective of $\gamma$. In other words, the conditions of Proposition 3.2(b) are satisfied provided that the shot has a tail decaying faster than logarithmically.

Example 3.3. (Benktander distributions.) We first consider the so-called Benktander distribution of type I (see, for instance, Embrechts et al. (1997)):

$$
\bar{B}(u)=(1+2(\delta / \alpha) \ln u) \exp \left\{-\left(\delta(\ln u)^{2}+(\alpha+1) \ln u\right)\right\}, \quad u \geq 1, \alpha, \delta>0 .
$$

We have $B \in \operatorname{MDA}(\Lambda), B_{0} \in \&$, and

$$
e(u)=\frac{u}{\alpha+2 \delta \ln u} \quad \text { for all } u \geq 1
$$

(see Embrechts et al. (1997)). In particular, $e(u)$ is eventually nondecreasing and condition (3.9) is satisfied for all $\gamma>1$. Hence, the conditions of the proposition are met if (3.8) holds for some $\gamma>1$. Again, this does not depend on $\gamma$, and is equivalent to $\bar{F}(u)=o(1 / \ln u)$.

Finally, assume that $B(\cdot)$ is a Benktander distribution of type II (see, for instance, Embrechts et al. (1997)) of the form

$$
\bar{B}(u)=\mathrm{e}^{\alpha / \delta} u^{-(1-\delta)} \exp \left\{-\frac{\alpha}{\delta} u^{\delta}\right\}, \quad u \geq 1,0<\alpha<1,0<\delta<1 .
$$

In this case $B \in \operatorname{MDA}(\Lambda), B_{0} \in \delta$, and $e(u)=u^{1-\delta} / \alpha, u \geq 1$ (see, for instance, Embrechts et al. (1997)). Therefore, $e(u)$ is nondecreasing and condition (3.9) is met for all $\gamma>1 /(1-\delta)$. As (3.8) can be rewritten as $\bar{F}(u)=o\left(u^{-\gamma \delta}\right)$, the conditions of the proposition are satisfied if this holds for some $\gamma>1 /(1-\delta)$. This is the case if $F$ is either light tailed or has a regularly varying tail of index $-\beta, \beta>\delta /(1-\delta)$.

Proof of Proposition 3.1. In view of (3.3), it only remains to prove the corresponding lower bound. By (3.6), we have

$$
\liminf _{u \rightarrow \infty} \frac{\psi(u)}{\bar{B}_{0}(u)} \geq \lim _{u \rightarrow \infty} \frac{\check{\Psi}_{a}((u+c a) / F(a))}{\bar{B}_{0}((u+c a) / F(a))} \lim _{u \rightarrow \infty} \frac{\bar{B}_{0}((u+c a) / F(a))}{\bar{B}_{0}(u)} .
$$

Now, since $\bar{B} \in \mathcal{R}(-\alpha-1)$, it follows from Karamata's theorem (see, for instance, Embrechts et al. (1997)) that $\overline{B_{0}} \in \mathcal{R}(-\alpha)$. Hence, by the definition of a regularly varying function,

$$
\lim _{u \rightarrow \infty} \frac{\bar{B}_{0}((u+c a) / F(a))}{\bar{B}_{0}(u)}=F(a)^{\alpha}
$$


Since $F(a) \leq 1$ for all $a$, it follows from the net profit condition that $\rho(a):=F(a) \mu / c v<1$. Hence, by the classical ruin estimate,

$$
\lim _{u \rightarrow \infty} \frac{\check{\Psi}_{a}((u+c a) / F(a))}{\bar{B}_{0}((u+c a) / F(a))}=\frac{\rho(a)}{1-\rho(a)} .
$$

Substituting (3.11) and (3.12) into (3.10) yields

$$
\liminf _{u \rightarrow \infty} \frac{\psi(u)}{\bar{B}_{0}(u)} \geq \frac{\rho(a) F(a)^{\alpha}}{1-\rho(a)} \quad \text { for all } a>0 .
$$

Letting $a$ tend to $\infty$, we notice that $F(a)$ tends to 1 and that $\rho(a)$ tends to $\rho$, which yields the desired lower bound and, thus, the claim of the proposition.

Proof of Proposition 3.2. For part (a), note that if $F$ has compact support then there is an $a<\infty$ such that $F(a)=1$. Consequently, from (3.10) we obtain

$$
\liminf _{u \rightarrow \infty} \frac{\psi(u)}{\bar{B}_{0}(u)} \geq \lim _{u \rightarrow \infty} \frac{\check{\Psi}_{a}(u+c a)}{\bar{B}_{0}(u+c a)} \lim _{u \rightarrow \infty} \frac{\bar{B}_{0}(u+c a)}{\bar{B}_{0}(u)} .
$$

The first limit in the product above equals $\rho /(1-\rho)$ by the classical ruin estimate, while the second limit equals 1 by the long tail property of subexponential distributions (see, for instance, Embrechts et al. (1997)). Thus, we obtain a lower bound on $\psi(u)$ matching the asymptotic upper bound in (3.3) and equal to the limit in (3.7), as claimed.

Next we turn to the proof of part (b). We can generalize (3.10) to let $a$ depend on $u$, that is, to have $a \equiv a(u)$. Also, note that, for any $x>0$ and $a \leq a(u), \check{\Psi}_{a}(x) \leq \check{\Psi}_{a(u)}(x)$. This is obvious on recalling that $\breve{\Psi}_{a}(x)$ denotes the ruin probability subject to initial capital $x$ and premium rate $c / F(a)$, as the ruin probability is nonincreasing in the premium rate. In other words,

$$
\psi(u) \geq \check{\Psi}_{a(u)}\left(\frac{u+c a(u)}{F(a(u))}\right) \geq \check{\Psi}_{a}\left(\frac{u+c a(u)}{F(a(u))}\right) .
$$

Therefore, we can rewrite (3.10) as

$$
\liminf _{u \rightarrow \infty} \frac{\psi(u)}{\bar{B}_{0}(u)} \geq \liminf _{u \rightarrow \infty} \frac{\check{\Psi}_{a}((u+c a(u)) / F(a(u)))}{\bar{B}_{0}((u+c a(u)) / F(a(u)))} \liminf _{u \rightarrow \infty} \frac{\bar{B}_{0}((u+c a(u)) / F(a(u)))}{\bar{B}_{0}(u)}
$$

for any $a>0$. Hence, using the classical ruin estimate applied to $\check{\Psi}_{a}$,

$$
\liminf _{u \rightarrow \infty} \frac{\psi(u)}{\bar{B}_{0}(u)} \geq \frac{\rho(a)}{1-\rho(a)} \liminf _{u \rightarrow \infty} \frac{\bar{B}_{0}((u+c a(u)) / F(a(u)))}{\bar{B}_{0}(u)} \quad \text { for all } a>0 .
$$

We now use a representation of $\bar{B}_{0}$ for $B \in \operatorname{MDA}(\Lambda)$ given in Asmussen and Klüppelberg (1996):

$$
\bar{B}_{0}(u)=\exp \left\{-\int_{0}^{u} \frac{1}{e(t)} \mathrm{d} t\right\}, \quad u>0 .
$$

It follows that

$$
\frac{\bar{B}_{0}((u+c a(u)) / F(a(u)))}{\bar{B}_{0}(u)}=\exp \left\{-\int_{u}^{(u+c a(u)) / F(a(u))} \frac{1}{e(t)} \mathrm{d} t\right\} .
$$


By the assumption of Proposition 3.2(b), $e(t) \sim g(t)$ for a function $g(\cdot)$ which is eventually nondecreasing. Hence, for all $\varepsilon>0$ we have

$$
\liminf _{u \rightarrow \infty} \frac{\bar{B}_{0}((u+c a(u)) / F(a(u)))}{\bar{B}_{0}(u)} \geq \liminf _{u \rightarrow \infty} \exp \left\{-\left(\frac{u+c a(u)}{F(a(u))}-u\right) \frac{1+\varepsilon}{g(u)}\right\} .
$$

Since $B \in \operatorname{MDA}(\Lambda)$ and $B_{0} \in \delta$, the mean excess function $e(u)$ goes to $\infty$ as $u$ goes to $\infty$ (see Goldie and Resnick (1988)). Let $\gamma>0$ be such that (3.8) and (3.9) hold, and take $a(u)=u^{1 / \gamma}$. Then

$$
\left(\frac{u+c a(u)}{F(a(u))}-u\right) \frac{1}{g(u)}=\frac{u \bar{F}\left(u^{1 / \gamma}\right)}{g(u) F\left(u^{1 / \gamma}\right)}+\frac{c u^{1 / \gamma}}{g(u) F\left(u^{1 / \gamma}\right)} \rightarrow 0 \quad \text { as } u \rightarrow \infty,
$$

since $g(u) \sim e(u)$. Therefore, by (3.14) and the fact that $\bar{B}_{0}(\cdot)$ is nonincreasing, we have

$$
\lim _{u \rightarrow \infty} \frac{\bar{B}_{0}\left(\left(u+c u^{1 / \gamma}\right) / F\left(u^{1 / \gamma}\right)\right)}{\bar{B}_{0}(u)}=1
$$

whenever $\gamma$ satisfies (3.8) and (3.9). Substituting this into (3.13) yields

$$
\liminf _{u \rightarrow \infty} \frac{\psi(u)}{\bar{B}_{0}(u)} \geq \frac{\rho(a)}{1-\rho(a)}
$$

for all $a>0$. Now, by letting $a \rightarrow \infty$, and noting that $\rho(a) \rightarrow \rho$, we obtain

$$
\liminf _{u \rightarrow \infty} \frac{\psi(u)}{\bar{B}_{0}(u)} \geq \frac{\rho}{1-\rho} .
$$

Combined with the upper bound in (3.3), this yields (3.7).

\subsection{The finite-horizon case}

Throughout this section we assume that $\left\{T_{n}\right\}_{n \geq 1}$, with $T_{0}=0$, is a homogeneous Poisson process with intensity $v^{-1}$. The following proposition holds.

Proposition 3.3. (a) If $\bar{B} \in \mathcal{R}(-\alpha-1), \alpha>0$, then

$$
\lim _{u \rightarrow \infty} \frac{\psi(u, u T)}{\psi(u)}=1-(1+(1-\rho) T)^{-\alpha} .
$$

(b) Suppose that the assumptions of Proposition 3.2(b) are satisfied for some $\gamma>1$ and with the function $g(\cdot)$ being regularly varying at infinity. Then

$$
\lim _{u \rightarrow \infty} \frac{\psi(u, e(u) T)}{\psi(u)}=1-\mathrm{e}^{-(1-\rho) T} .
$$

Remark 3.1. Note that $e(u) \sim g(u)$ with $g(\cdot)$ regularly varying at infinity is satisfied in all the examples considered above.

Remark 3.2. Proposition 3.3 says that, starting with initial capital $u$ and conditional on ruin occurring, the time to ruin scales like $u$ or $e(u)$ under the assumptions of part (a) or part (b), respectively. More precisely, the time to ruin divided by $u$ converges in distribution to a Pareto random variable under the assumptions of part (a), while the time to ruin divided by $e(u)$ converges in distribution to an exponential random variable under the assumptions of part (b). 
Proof of Proposition 3.3. We first show the upper bounds for (3.16) and (3.17). Respectively denote by $\Psi(u, T)$ and $\Psi(u)$ the finite-horizon and the infinite-horizon ruin probabilities relative to the classical risk process $\{C(t)\}_{t \geq 0}$ defined in (3.1). By Corollary 1.6 of Asmussen and Klüppelberg (1996) (see also Asmussen (2000, p. 275-279)), we have

$$
\lim _{u \rightarrow \infty} \frac{\Psi(u, u T)}{\Psi(u)}=1-(1+(1-\rho) T)^{-\alpha}
$$

if $\bar{B} \in \mathcal{R}(-\alpha-1)$ and

$$
\lim _{u \rightarrow \infty} \frac{\Psi(u, e(u) T)}{\Psi(u)}=1-\mathrm{e}^{-(1-\rho) T}
$$

if $B \in \operatorname{MDA}(\Lambda)$ and $B_{0} \in$ \&. By Propositions 3.1 and 3.2, $\psi(u) \sim \Psi(u)$ under the assumptions of this proposition. Since (3.2) implies that $\psi(u, \tilde{g}(u) T) \leq \Psi(u, \tilde{g}(u) T)$ for all $u$ and all nonnegative functions $\tilde{g}(\cdot)$, the upper bounds follow by (3.18) and (3.19).

It remains to prove the matching lower bounds. We first show part (a). For each $a>0$, respectively denote by $\check{\Psi}_{a}(u, T)$ and $\check{\Psi}_{a}(u)$ the finite-horizon and the infinite-horizon ruin probabilities relative to the risk process $\left\{\check{C}_{a}(t)\right\}_{t \geq 0}$ defined in (3.4). As in the proof of Proposition 3.1, we obtain (3.5), which yields, for each $a>0$,

$$
\psi(u, u T) \geq \check{\Psi}_{a}\left(\frac{u+c a}{F(a)}, u T-a\right) \text { for all } u>0,
$$

where $\check{\Psi}_{a}(u, t)$ is defined to equal 0 if $t<0$. Since $u-(a / T) \sim u+c a$ as $u \rightarrow \infty$, by (3.20) it follows that for any $\varepsilon>0$ there exists a $\bar{u} \equiv \bar{u}(\varepsilon)$ such that

$$
\psi(u, u T) \geq \check{\Psi}_{a}\left(\frac{u+c a}{F(a)},(u+c a)(1-\varepsilon) T\right) \text { for all } u \geq \bar{u} \text { and all } a>0 .
$$

Since (3.18) also holds with $\check{\Psi}_{a}$ in place of $\Psi$ (the former is simply the ruin probability for the risk process modified to have premium rate $c / F(a)$ ), we obtain

$$
\lim _{u \rightarrow \infty} \frac{\check{\Psi}_{a}((u+c a) / F(a),(u+c a)(1-\varepsilon) T)}{\check{\Psi}_{a}((u+c a) / F(a))}=1-(1+(1-\rho(a))(1-\varepsilon) T F(a))^{-\alpha},
$$

where $\rho(a)=F(a) \mu / c v=\rho F(a)$. Arguing as in the proof of Proposition 3.1, we have

$$
\lim _{u \rightarrow \infty} \frac{\check{\Psi}_{a}((u+c a) / F(a))}{\psi(u)}=\frac{\rho(a) F(a)^{\alpha}}{1-\rho(a)}\left(\frac{\rho}{1-\rho}\right)^{-1} .
$$

The matching lower bound follows upon combining (3.21), (3.22), and (3.23), letting $\varepsilon$ tend to 0 , and letting $a$ tend to $\infty$.

Finally, we show part (b). It follows from (3.5) that, for an arbitrary positive function $a(\cdot)$, an arbitrary constant $a>0$, and all $u>0$ such that $a(u) \geq a$,

$$
\psi(u, e(u) T) \geq \check{\Psi}_{a(u)}\left(\frac{u+c a(u)}{F(a(u))}, e(u) T-a(u)\right) \geq \check{\Psi}_{a}\left(\frac{u+c a(u)}{F(a(u))}, e(u) T-a(u)\right) .
$$

Recall that $e(\cdot)$ was assumed to be asymptotically equivalent to a regularly varying function $g(\cdot)$. Let $\beta$ denote the index of variation of $g$ at infinity, let $\gamma>1$ be such that (3.8) and (3.9) hold, and take $a(u)=u^{1 / \gamma}$. Then

$$
\lim _{u \rightarrow \infty} \frac{e((u+c a(u)) / F(a(u)))}{e(u)}=\lim _{u \rightarrow \infty}\left(\frac{u+c u^{1 / \gamma}}{u F\left(u^{1 / \gamma}\right)}\right)^{\beta}=1,
$$


where we have used the fact that $\gamma>1$ to obtain the second equality. Since $u^{1 / \gamma}=o(e(u))$, by (3.9), it follows that

$$
\lim _{u \rightarrow \infty} \frac{e(u) T-a(u)}{e((u+c a(u)) / F(a(u)))}=T .
$$

Therefore, by (3.19), for all $\varepsilon>0$ we obtain

$$
\liminf _{u \rightarrow \infty} \frac{\check{\Psi}_{a}((u+c a(u)) / F(a(u)), e(u) T-a(u))}{\check{\Psi}_{a}((u+c a(u)) / F(a(u)))} \geq 1-\mathrm{e}^{-(1-\varepsilon)(1-\rho(a)) T} .
$$

Combining the above with (3.24) and letting $\varepsilon$ decrease to 0 yields

$$
\liminf _{u \rightarrow \infty} \frac{\psi(u, e(u) T)}{\check{\Psi}_{a}((u+c a(u)) / F(a(u)))} \geq 1-\mathrm{e}^{-(1-\rho(a)) T} .
$$

It remains only to compare the denominator of the left-hand side with $\Psi(u)$. Using the classical ruin estimate, we have

$$
\liminf _{u \rightarrow \infty} \frac{\check{\Psi}_{a}((u+c a(u)) / F(a(u))}{\Psi(u)}=\frac{\rho(a)}{1-\rho(a)} \frac{1-\rho}{\rho} \liminf _{u \rightarrow \infty} \frac{\bar{B}_{0}((u+c a(u)) / F(a(u)))}{\bar{B}_{0}(u)} .
$$

If we take $a(u)=u^{1 / \gamma}$ with a $\gamma>1$ that satisfies the assumptions of Proposition 3.2(b), then (3.15) holds. Therefore,

$$
\liminf _{u \rightarrow \infty} \frac{\check{\Psi}_{a}\left(\left(u+c u^{1 / \gamma}\right) / F\left(u^{1 / \gamma}\right)\right)}{\Psi(u)}=\frac{\rho(a)}{1-\rho(a)} \frac{1-\rho}{\rho} .
$$

By substituting this into (3.25) and using the asymptotic equivalence of $\psi(u)$ and $\Psi(u)$ established in Proposition 3.2, we obtain

$$
\liminf _{u \rightarrow \infty} \frac{\psi(u, e(u) T)}{\psi(u)} \geq \frac{\rho(a)}{1-\rho(a)} \frac{1-\rho}{\rho}\left(1-\mathrm{e}^{-(1-\rho(a)) T}\right)
$$

for all $a>0$. By now letting $a$ tend to $\infty$, and noting that $\rho(a)$ tends to $\rho$, we obtain the lower bound

$$
\liminf _{u \rightarrow \infty} \frac{\psi(u, e(u) T)}{\psi(u)} \geq 1-\mathrm{e}^{-(1-\rho) T} .
$$

Combining this with the upper bound established earlier completes the proof of the proposition.

\section{Appendix A.}

In this section we obtain logarithmic asymptotics for the infinite-horizon ruin probability when the claim sizes have exponential tails and the claim arrival process is Poisson. As in Brémaud (2000), we consider the more general form of the risk process with delayed claims, namely

$$
S(t)=\sum_{n \geq 1} H\left(t-T_{n}, Z_{n}\right) \mathbf{1}_{(0, t]}\left(T_{n}\right)-c t .
$$

Here $T_{0}=0$ and $\left\{T_{n}\right\}_{n \geq 1}$ are the points of a Poisson process of rate $\lambda$, independent of the independent, identically distributed sequence of marks $\left\{Z_{n}\right\}_{n \geq 1}$ taking values in a measure space $(E, \mathcal{E})$, and $H: \mathbb{R}_{+} \times E \rightarrow[0, \infty)$ is a measurable function such that $H(\cdot, z)$ is 
nondecreasing and càdlàg (right continuous with left limits) for all $z \in E$. We define $H(\infty, z)=$ $\lim _{t \rightarrow \infty} H(t, z)$, noting that the limit exists but may be infinite. The ruin probability $\psi(u)$ for $u>0$ is as defined in (1.2).

We compare the risk process $S(\cdot)$ with the classical risk process $C(\cdot)$ defined by

$$
C(t)=\sum_{n \geq 1} H\left(\infty, Z_{n}\right) \mathbf{1}_{(0, t]}\left(T_{n}\right)-c t,
$$

with corresponding ruin probability $\Psi(u)=\mathrm{P}\left(\sup _{t \geq 0} C(t)>u\right)$. We are interested in the case where the claim size distribution has exponentially decaying tails, that is, $\mathrm{E}\left[\exp \left\{\theta H\left(\infty, Z_{1}\right)\right\}\right]$ is finite for $\theta$ in a neighborhood of 0 . If Cramér's condition is satisfied, that is, if

$$
\text { there exists an } w>0 \text { such that } \lambda\left(\mathrm{E}\left[\mathrm{e}^{w H\left(\infty, Z_{1}\right)}\right]-1\right)-c w=0,
$$

then we have the classical result

$$
\lim _{u \rightarrow \infty} \frac{1}{u} \log \Psi(u)=-w .
$$

It was shown in Brémaud (2000) that $\psi(u)$ satisfies the same logarithmic asymptotics, that is,

$$
\lim _{u \rightarrow \infty} \frac{1}{u} \log \psi(u)=-w .
$$

We now give an alternative proof of this result. Since $\psi(u) \leq \Psi(u)$ for all $u>0$, it suffices to prove the asymptotic lower bound. To this end, observe that, for all $a>0$,

$$
S(t) \geq \sum_{n \geq 1} H\left(a, Z_{n}\right) \mathbf{1}_{(0, t-a]}\left(T_{n}\right)-c(t-a)-c a=C_{a}(t-a)-c a,
$$

where $C_{a}(\cdot)$ is defined analogously to $C(\cdot)$, but with $H\left(\infty, Z_{1}\right)$ replaced with $H\left(a, Z_{1}\right)$. Define $\Psi_{a}$ to be the ruin probability associated with the risk process $C_{a}(\cdot)$. It follows from the above that

$$
\psi(u) \geq \Psi_{a}(u+c a) \text { for all } u>0 \text { and all } a>0 .
$$

Also, by the Cramér-Lundberg theorem, for each $a>0$ we have

$$
\lim _{u \rightarrow \infty} \frac{1}{u} \log \Psi_{a}(u)=-w_{a},
$$

where $w_{a}$ is the unique positive solution to

$$
\lambda\left(\mathrm{E}\left[\mathrm{e}^{w_{a} H\left(a, Z_{1}\right)}\right]-1\right)-c w_{a}=0,
$$

if one exists; otherwise $w_{a}=\infty$. Since $H\left(a, Z_{1}\right)$ increases to $H\left(\infty, Z_{1}\right)$ as $a \rightarrow \infty$, it readily follows that $w_{a}$ decreases to $w$. Therefore, it is immediate from (A.2) and (A.3) that

$$
\liminf _{u \rightarrow \infty} \frac{1}{u} \log \psi(u) \geq-\limsup _{a \rightarrow \infty} w_{a}=-w .
$$

Combined with the upper bound established earlier, this completes the proof of (A.1).

Finally, we remark that the extension of the results to the case when $\left\{T_{n}\right\}_{n \geq 1}$ constitute a renewal process is straightforward. The results can also be extended to allow certain kinds of dependence between $T_{n}$ and $Z_{n}$, by following the methods of Albrecher and Teugels (2006). As the heavy tail case is the main focus of this paper, we do not pursue these extensions here. 


\section{Acknowledgement}

The authors thank the referee for helpful suggestions on the presentation of the results, and for bringing recent references to their attention.

\section{References}

Albrecher, H. And Asmussen, S. (2006). Ruin probabilities and aggregate claims distributions for shot noise Cox processes. Scand. Actuarial J. 2006, 86-110.

Albrecher, H. ANd Teugels, J. L. (2006). Exponential behavior in the presence of dependence in risk theory. J. Appl. Prob. 43, 257-273.

Asmussen, S. (2000). Ruin Probabilities. World Scientific, Singapore.

Asmussen, S. AND KlüPPElberg, C. (1996). Large deviations results for subexponential tails, with applications to insurance risk. Stoch. Process. Appl. 64, 103-125.

Asmussen, S., Schmidli, H. And Schmidt, V. (1999). Tail probabilities for non-standard risk and queueing processes with subexponential jumps. Adv. Appl. Prob. 31, 422-447.

Brémaud, P. (2000). An insensitivity property of Lundberg's estimate for delayed claims. J. Appl. Prob. 37, 914-917.

Embrechts, P. and Veraverbeke, N. (1982). Estimates for the probability of ruin with special emphasis on the possibility of large claims. Insurance Math. Econom. 1, 55-72.

Embrechts, P., KlüPPelberg, C. AND Mikosch, T. (1997). Modelling Extremal Events for Insurance and Finance. Springer, Berlin.

Goldie, C. M. And Resnick, S. (1988). Distributions that are both subexponential and in the domain of attraction of an extreme-value distribution. Adv. Appl. Prob. 20, 706-718.

KlÜPPElberg, C. AND Mikosch, T. (1995a). Delay in claim settlement and ruin probability approximations. Scand. Actuarial J. 1995, 154-168.

KlÜPPElberg, C. AND MiKosch, T. (1995b). Explosive Poisson shot noise processes with applications to risk reserves. Bernoulli 1, 125-147.

Klüppelberg, C., Mikosch, T. And Schärf, A. (2003). Regular variation in the mean and stable limits for Poisson shot noise. Bernoulli 9, 467-496.

Macci, C., Stabile, G. And Torrisi, G. L. (2005). Lundberg parameters for non standard risk processes. Scand. Actuarial J. 2005, 417-432.

Mikosch, T. AND NAGAEv, V. (1998). Large deviations of heavy-tailed sums with applications in insurance. Extremes 1, 81-110.

Rolski, T., Schmidli, H., Schmidt, V. And Teugels, J. L. (1999). Stochastic Processes for Insurance and Finance. John Wiley, Chichester.

Teugels, J. L. And Veraverbeke, N. (1973). Cramér-type estimates for the probability of ruin. CORE Discussion Paper 7316, Université catholique de Louvain.

Veraverbeke, N. (1977). Asymptotic behaviour of Wiener-Hopf factors of a random walk. Stoch. Process. Appl. 5, 27-37.

Waters, H. R. and Papatriandafylou, A. (1985). Ruin probabilities allowing for delay in claims settlement. Insurance Math. Econom. 4, 113-122.

Yuen, K. C., Guo, J. And Ng, K. W. (2005). On ultimate ruin in a delayed-claims risk model. J. Appl. Prob. 42, 163-174. 